\title{
Integrated management of HIV/NCDs: knowledge, attitudes, and practices of health care workers in Gaborone, Botswana
}

\author{
Tiny Masupe ${ }^{1}$, Yohana Mashallaa ${ }^{2,5}$, Esther Seloilwe ${ }^{3}$, Harun Jibril ${ }^{4}$, Heluf Medhin ${ }^{4}$
}

1. Department of Family Medicine and Public Health, Faculty of Medicine, University of Botswana.

2. Department of Biomedical Sciences, Faculty of Medicine, University of Botswana.

3. School of Nursing, Faculty of Health Sciences, University of Botswana.

4. Ministry of Health, Government of Botswana.

5. Department of Health Studies, University of South Africa.

\begin{abstract}
Background: The epidemiologic transition and double disease burden from chronic infections and Non-communicable diseases (NCDs) worldwide requires re-engineering of healthcare delivery systems. Healthcare workers (HCWs) need to adapt to new integrated disease management approaches and change from current disease-specific management.

Objectives: The study aimed to determine HCWs knowledge, capacity and skills for management of NCDs among HIV patients and their attitudes towards integrated HIV/NCDs disease management approaches for future clinical practice.

Methods: Descriptive cross-sectional survey among HCWs attending to HIV patients at selected government facilities.

Results: One hundred out of 105 responses were analysed. Only $6 \%$ could fully define NCDs. Awareness levels of NCDs were high: Diabetes and hypertension 98\%; cancer 96\%; cardiovascular diseases $86 \%$. However, $11.8 \%$ and $58 \%$ classified HIV and malaria respectively as NCDs. Most respondents $(88 \%)$ believe that integrating HIV/NCDs care would be good use of resources while $62 \%$ disagreed with current separate facility management of HIV patients with NCDs. Over $60 \%$ routinely screened HIV patients for NCDs risk factors: Smoking (87.2\%), alcohol (90.8\%), diet (84.9\%) and physical activity $(73.5 \%)$.

Conclusion: There were gaps in detailed knowledge on NCDs, but positive attitude towards routine primary care integrated HIV/NCDs management, showing likely support for implementation of such policy.

Keywords: Non-communicable diseases, knowledge, attitude, HIV, integration.

DOI: https://dx.doi.org/10.4314/ahs.v19i3.3

Cite as: Masupe T, Mashalla Y, Seloilwe E, Jibril H, Medhin H. Integrated management of HIV/NCDs: knowledge, attitudes, and practices of health care workers in Gaborone, Botswana. Afri Health Sci. 2019;19(3):2312-2323. bttps:/ / dx.doi.org/10.4314/ abs.v19i3.3
\end{abstract}

\section{Background}

The World Health Organisation estimates that more than 70 million people have been infected with HIV and 35 million people have died since the 1980s. Globally, 36.7 million people were living with HIV at the end of 2015 with an estimated $0.8 \%$ of those being adults aged $15-$
Corresponding author:
Tiny Masupe;
Department of Family Medicine
and Public Health, University of Botswana.
Tel: +2673554839
Email: masupet@ub.ac.bw

49 years. Sub-Saharan Africa remains most affected with nearly 1 in every 25 adults (4.4\%) living with HIV and accounting for nearly $70 \%$ global HIV prevalence ${ }^{1}$.

Botswana is among the most affected countries by HIV and AIDS with HIV prevalence estimated at 18\% (BIAS IV, 2016). The MASA national ART program reports that by December 2014, 247,947 (11\%) of Batswana adults and children were on $\mathrm{ART}^{2}$. In 2001, the average life expectancy in Botswana was estimated at 46.5 years attributed to the impact of Human Immunodeficiency Virus and Acquired Immune Deficiency syndrome (HIV/AIDS) ${ }^{3}$. In 2013 however, the average life expectancy at birth for Botswana had risen to 64 years for males and 67 years

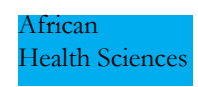

(C) 2019 Masupe et al. Licensee African Health Sciences. This is an Open Access article distributed under the terms of the Creative commons Attribution License (https://creativecommons.org/licenses/BY/4.0), which permits unrestricted use, distribution, and reproduction in any medium, provided the original work is properly cited. 
for females ${ }^{4}$. This increase was attributed to improved access to life saving HIV treatment. Botswana launched the treat all strategy on 3rd June 2016. According to the Botswana combination prevention project study in 2016, $29 \%$ of those surveyed were HIV positive and out of those $83 \%$ already knew their status, $87.4 \%$ were on ART and $73 \%$ were virologically suppressed suggesting that Botswana was very close to attaining the 90-90-90 ART and virologic suppression goals by 2020 . With the advent of High Active Anti-Retroviral Treatment (HAART), HIV has become a chronic medical condition with those on treatment living longer and developing other chronic non-communicable diseases (NCDs) some of which are related to the side effects of antiretroviral medications and some due to the chronic HIV infection itself $f^{5-7}$.

WHO further estimates that in the next ten years, the global NCDs burden will increase by $17 \%$, and in Africa region by $27 \%{ }^{6}$. Over $80 \%$ of cardiovascular diseases (CVD) and diabetes deaths, 90\% of chronic obstructive pulmonary diseases (COPD) deaths and about $70 \%$ of all cancer deaths occur in developing countries ${ }^{7}$. Botswana is no exception to the growing prevalence of NCDs worldwide $^{10,11}$. Between 1980 and 1998 Botswana has recorded 5-fold and 2-fold increases in health service utilisation due to hypertension and diabetes respectively and the STEPS survey 2007 identified 35\% of those aged 20 69 years as having risk of developing any type of NCD? The Botswana National Health Statistics reports between 2005 and 2009 have indicated gradual increases in the number of admissions and deaths due to NCDs nationally ${ }^{13,14}$ therefore, NCDs are a barrier to development ${ }^{11}$.

Currently, treatment guidelines on the management of all diseases are available in the Botswana Primary Care Guidelines which cover the management of diabetes, hypertension, cardiovascular diseases, mental health and non- HIV related cancers in a primary care setting. The NCD management model remains that of addressing acute presentations of NCDs rather than long term risk factor management. This limitation is noted as one of the weaknesses in the strength, weakness, opportunity and threat (SWOT) analysis for the Botswana prevention and control of NCDs strategic plan 2011-2016

The recognition of multiple-morbidities of chronic diseases among HIV infected and the increasing patient workload due to co-morbidities of HIV, infectious chronic diseases (ICDs) and NCDs calls for a more coordinated patient-centred approach to disease management in the country rather than the current disease specific focus ${ }^{16,17}$ and to learn from the experiences of countries that have started to integrate HIV management with other chronic diseases $^{18-21}$. In December 2016, the Botswana MoHW started a review of the essential primary care package due to recognition that at the time of the conception of the package in 2012, NCDs had not become prevalent at current levels $^{13}$. The Botswana health system needs to be prepared for the inevitability of integrated management of HIV and other chronic diseases and the requirements that its healthcare workers will have to adapt to, for such a policy to be implemented successfully.

Botswana health system is unlikely to successfully implement routine integrated HIV and NCDs management care unless its healthcare workers are knowledgeable and capacitated on combined NCD/HIV disease management models. The main aim of this study was therefore to assess the knowledge of healthcare workers on NCDs, the link between NCDs and HIV as well as their attitudes and practices towards integrated management of NCDs and HIV in Botswana so that we contribute to advising policy makers on pre-emptive steps to take to prepare for integrated HIV/NCDs management. Specific objectives were i) To determine the knowledge of HCWs on the four common NCDs (diabetes, hypertension, cancers, CVDs) ii) To assess the HCWs knowledge of common NCDs among HIV patients use iii) To identify the current practices of HCWs pertaining to screening for risk factors for NCDs during the routine HIV consultation process in public health facilities in the Gaborone health district and iv) to describe HCWs attitudes towards integrated management of HIV/NCDs at primary care level.

\section{Methods}

\section{Research design}

This was a descriptive cross-sectional survey of HCWs providing primary care for HIV patients in Gaborone health district clinics. Clinics were chosen because in Botswana they are the first patient point of contact with the health system and are therefore a focal point for primary care services. Gaborone District Health Management Team (GDHMT) currently provides oversight to 30 clinics and employs 500 nurses and 43 doctors.

\section{Data collection methods}

The sampling frame was a list of all clinics under the GDHMT. Simple random sampling was used to select 
the clinics. Clinics with and without an Infectious Disease Control Center (IDCC) were included given that those without IDCC still provide general HIV services. Each selected clinic was contacted for permission to participate in the study. Data was collected from March to July 2015.

\section{Sample size}

The formula commonly used to estimate sample size for this study was as follows

$\mathrm{N}=$ sample size

$\mathrm{Z}=$ value corresponding to a given confidence level (1.96

for a confidence level of $95 \%$ )

$\mathrm{p}=$ percentage of the primary indicator expressed as a decimal (0.5 for this study)

$\mathrm{c}=$ standard error, expressed as a decimal (0.05 for this study).

All nurses and doctors providing HIV treatment and care services in the selected clinics were eligible for participation in the study. The calculated sample size was 384 but given the small numbers due to poor response rate, purposive sampling was used for the HCWs. Those selected met the inclusion criteria of adults aged 21 years or above; nurse or doctor working in the selected clinic, having provided care to HIV patients for a minimum of 6 months prior to the study; willingness to participate in the study and given informed consent. Those HCWs who were not able to give voluntary informed consent to participate in the study; had no HIV management training of any kind, not managed HIV patients in the last 12 months and below 21 years of age were excluded from the study

Measures employed to reduce bias in the research included random sampling of health facilities, ii) use of trained research assistants iii) self-administration of question- naires by participants to reduce potential social desirability effect iv) data analysis by a statistician who was not a member of the research team but with guidance from the research team.

An adapted pre-tested survey questionnaire based on the WHO Steps Survey Questionnaire was used to collect the data. Data was entered directly from the questionnaires onto excel spread sheet and cleaned before analysis. Data analysis was done using SPSS statistical software package version 20. Descriptive statistics were calculated using measures of central tendency and simple percentages were reported. Cross-referencing of variables was also done. Results were reported using descriptive statistics.

\section{Ethical issues}

Written informed consent, privacy and confidentiality, voluntary participation and data anonymization at reporting were maintained in the study. The study received ethical clearance from the University of Botswana Institutional Review Board UB/IRB/1551 and MOHW. Permission to conduct the study at the various health care facilities was also granted by the GDHMT coordinator and the matron in charge of each clinic.

\section{Results}

\section{Demographic characteristics of respondents}

Demographic characteristics of the respondents are shown in Table 1. The total number of respondents was 105, out of which 100 were eligible for analysis. There were 80 females and 17 males and 3 undeclared genders. Majority (93\%) were nurses, 1 doctor, 4 other cadre and 2 with missing information on their designation. All respondent's major duties six months prior to the study included HIV care. 
Table 1: Demographic characteristics of the healthcare workers participating in the study

\begin{tabular}{|l|c|c|}
\hline \multicolumn{3}{|c|}{ Demographic characteristics of respondents N=100 } \\
\hline Variable & $\mathbf{N}$ & $\%$ \\
\hline \multicolumn{3}{|c|}{} \\
\hline Gender & 80 & 80 \\
Female & 17 & 17 \\
Male & 3 & 3 \\
\hline Not declared & & \\
\hline \multicolumn{3}{|l|}{} \\
\hline Designation & 93 & 93 \\
Nurse & 1 & 1 \\
Doctor & 4 & 4 \\
Other & 2 & 2 \\
Not declared & \\
\hline \multicolumn{2}{|l|}{} \\
\hline
\end{tabular}

\section{Healthcare workers awareness of NCDs}

Knowledge of HCWs on NCDs was assessed by asking them to define NCD and to indicate their awareness of NCDs. Only $6 \%$ and $66 \%$ of the respondents provided full definition and partial definition respectively of NCDs as diseases that are not passed from person to person, are of long duration and generally slow progression ${ }^{8}$. Less than half $(48 \%)$ stated they had heard about NCDs prior to this study while $28 \%$ had not heard about NCDs at all.

\section{Healthcare workers knowledge of NCDs}

Knowledge was assessed by HCWs ability to correctly identify NCDs from a list of diseases as presented in Table 2. High blood pressure and diabetes mellitus were identified correctly as NCDs by majority (97.8\%) followed by cancer $(95.6 \%)$, heart attack $(93.5 \%)$ depression $(88.9 \%)$ and road traffic injuries (69.0\%). Interestingly, $58 \%$ and $11.8 \%$ incorrectly classified malaria and HIV respectively as NCDs.

Table 2: HCW's ability to correctly identify NCDs from a list of diseases

\begin{tabular}{|l|c|c|c|c|c|}
\hline \multirow{2}{*}{$\begin{array}{c}\text { Which of the } \\
\text { following are NCDs? }\end{array}$} & & \multicolumn{2}{c|}{ Yes } & \multicolumn{2}{c|}{ No } \\
\cline { 2 - 6 } & N & Frequency & $\%$ & Frequency & $\%$ \\
\hline High Blood pressure & 92 & 90 & 97.8 & 2 & 2.2 \\
\hline Diabetes & 92 & 90 & 97.8 & 2 & 2.2 \\
\hline Road Traffic Injuries & 87 & 60 & 69 & 27 & 31 \\
\hline Heart Attack & 92 & 86 & 93.5 & 6 & 6.5 \\
\hline Cancer & 91 & 87 & 95.6 & 4 & 4.4 \\
\hline Malaria & 85 & 49 & 57.6 & 36 & 42.4 \\
\hline Depression & 90 & 80 & 88.9 & 10 & 11.1 \\
\hline Tuberculosis & 84 & 4 & 4.8 & 80 & 95.2 \\
\hline HIV & 85 & 10 & 11.8 & 75 & 88.2 \\
\hline
\end{tabular}




\section{Knowledge of risk factors for cardiovascular diseas-} es

The HCW's knowledge on CVDs was assessed by asking respondents to select "True, False or I do not know" from a list of risk factors for CVDs. Patients with high blood pressure are more likely to have stroke was recorded as "True" by $91 \%$ of the respondents. Majority had the knowledge that hypertension damages the heart and is a risk factor for CVDs. Being overweight was selected as "True" risk factor for CVD by majority (94\%); eating low salt diet and vegetables can prevent CVD was also selected as "True" by the majority (90\%). The HCWs also have the knowledge that cardiovascular diseases can be prevented. Responses are presented in the Table 3.

Table 3: HCWs knowledge of risk factors for cardiovascular diseases

\begin{tabular}{|c|c|c|c|c|c|}
\hline \multirow{2}{*}{ Knowledge } & \multirow[t]{2}{*}{$\mathbf{N}$} & \multicolumn{2}{|c|}{ Yes } & \multicolumn{2}{|c|}{ No } \\
\hline & & Frequency & $\%$ & Frequency & $\%$ \\
\hline $\begin{array}{l}\text { Is high blood pressure more likely to } \\
\text { cause stroke? }\end{array}$ & 93 & 91 & 91.0 & 2 & 2.0 \\
\hline $\begin{array}{l}\text { Can heart attack occur due to High blood } \\
\text { cholesterol? }\end{array}$ & 89 & 88 & 88.0 & 1 & 1.0 \\
\hline Can CVDs result in leg amputation? & 71 & 34 & 34.0 & 37 & 37.0 \\
\hline Hypertension does not damage the heart & 93 & 8 & 8.0 & 85 & 85.0 \\
\hline Can stroke cause Blindness? & 76 & 56 & 56.0 & 20 & 20.0 \\
\hline Hypertension is not a risk factor for CVDs & 89 & 6 & 6.0 & 83 & 83.0 \\
\hline $\begin{array}{l}\text { Can eating low salt diet and vegetables } \\
\text { prevent CVDs? }\end{array}$ & 90 & 90 & 90.0 & 0 & 0.0 \\
\hline $\begin{array}{l}\text { Can cardiovascular diseases be } \\
\text { prevented? }\end{array}$ & 92 & 91 & 91.0 & 1 & 1.0 \\
\hline $\begin{array}{l}\text { Is being overweight a risk factor for } \\
\text { CVDs? }\end{array}$ & 94 & 94 & 94.0 & 0 & 0.0 \\
\hline
\end{tabular}

\section{Healthcare workers' knowledge on commonly oc- curring cancers in Botswana}

The knowledge of HCWs on the common cancers ranked according to prevalence in Botswana was assessed by asking respondents to select from a list of cancers. Figure 1 shows that cervical cancer (57\%) and breast cancer (30\%) were selected as most common cancers while esophageal cancer was the lowest (1\%) and $8 \%$ did not respond to the question.
Healthcare workers' attitude towards NCDs as serious medical conditions

The attitude of HCWs towards NCDs was assessed by asking respondents to indicate the level of seriousness they associate with NCDs. Almost equal proportion 45\% and $46 \%$ rated NCDs as serious and extremely serious respectively (Figure 2). 


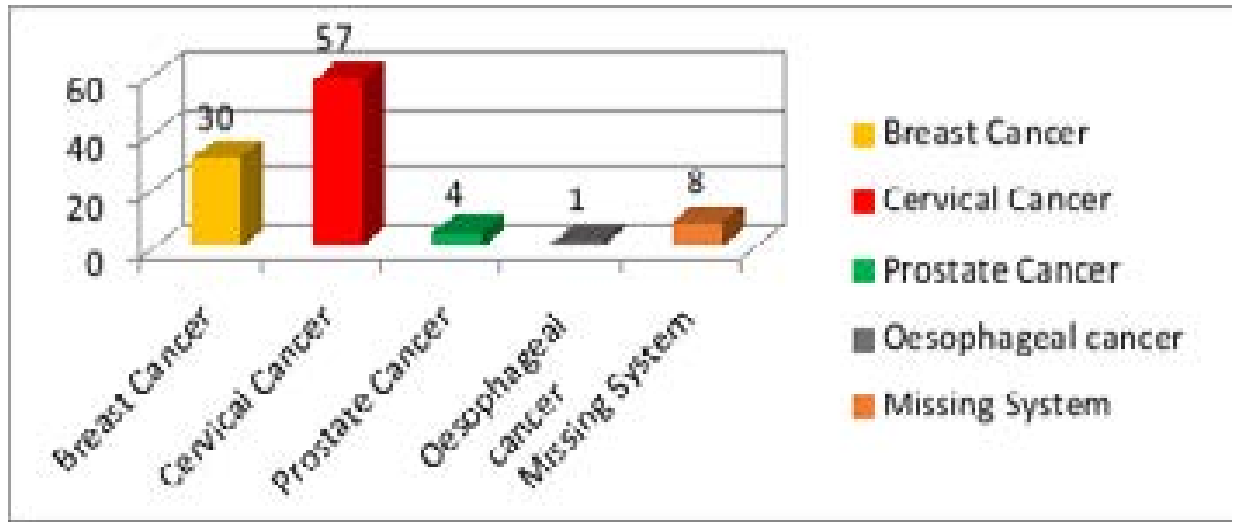

Figure 1: HCW knowledge of the commonly occurring cancers in Botswana

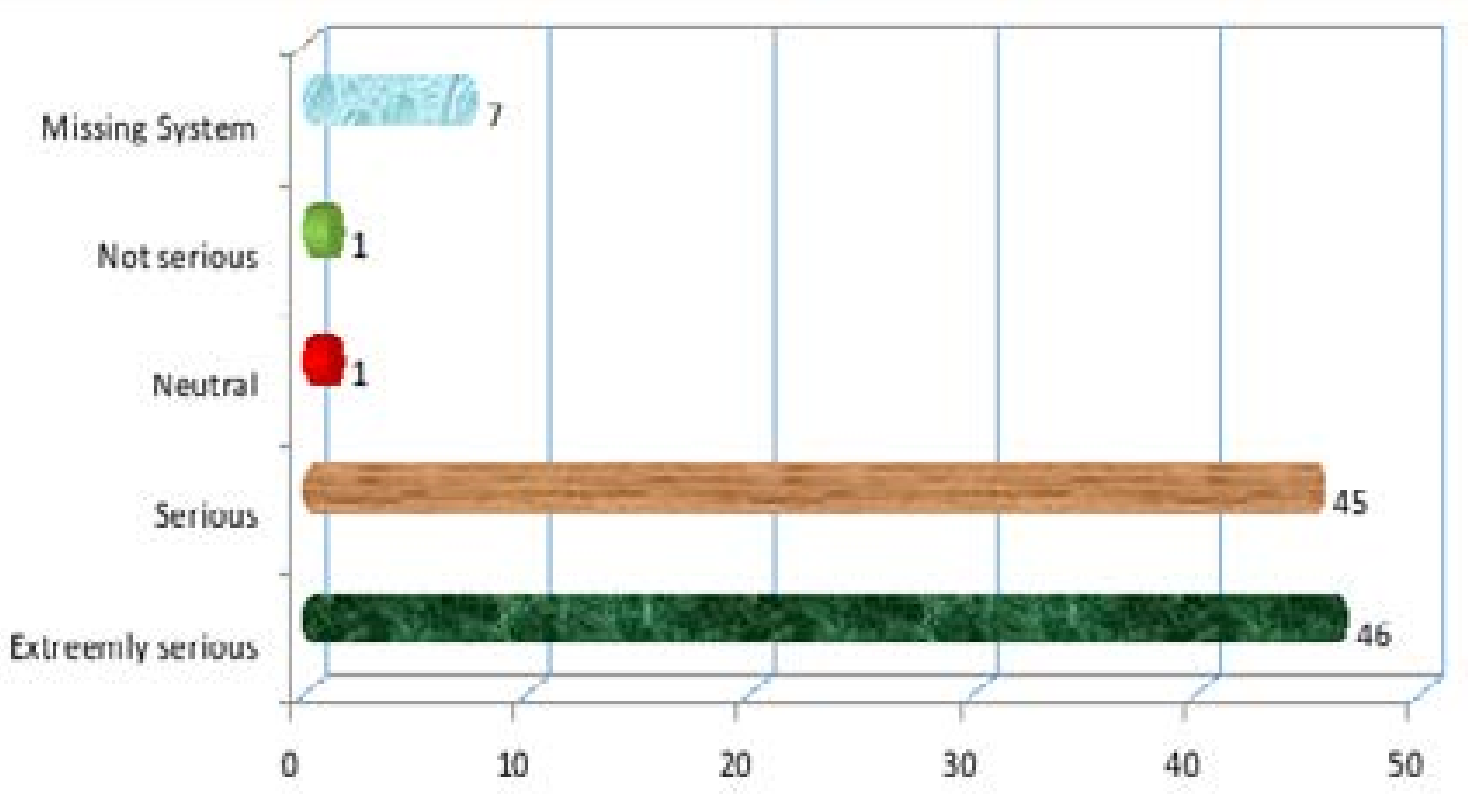

Figure 2: HCWs attitude towards the seriousness of NCDs.

Additionally, health care workers' reaction to their own diagnosis of NCD was explored. Just over half (53\%) HCWs indicated that they would be extremely concerned if diagnosed with NCD, 30\% would be concerned while $8 \%$ were neutral. The rest $(9 \%)$ did not respond to the question.

Healthcare workers knowledge of existence of NCDs among HIV infected patients

HCWs knowledge on prevalence of NCDs amongst HIV infected patients was assessed by asking them to indicate their level of agreement with the following statement: "NCDs are common among HIV patients". A total of $43 \%$ disagreed with the statement with $17 \%$ of those strongly disagreeing that NCDs are prevalent among HIV patients. However, over a third (35\%) agreed that NCDs are common among HIV patients but with only $9 \%$ agreeing strongly that NCDs are common among HIV patients. 
HCWs perceptions on prevalence of NCDs amongst HIV patients

The ability of the HCWs to correctly identify prevalent NCDs among HIV infected patients was assessed by asking them the question "Which of the following NCDs are common among those infected with HIV?". Results in table 4 below show that according to the HCWs, cervical cancer is the most common NCD among HIV patients (95.3\%), followed by high blood pressure (53.0\%) and breast cancer (50.6\%). Dyslipidaemia, heart attack and impaired glucose tolerance were considered less prevalent NCDs among HIV infected patients.

\section{Behaviours routinely screened for by HCWs during consultation with HIV patients}

The HCWs were asked to select behavioural risk factors that they frequently screen for among HIV patients during consultation. Table 5 shows that a large number of the interviewed HCWs routinely screen HIV patients for physical activity $(73.5 \%)$, alcohol intake (90.8\%), diet $(73 \%)$ and smoking $(87 \%)$ during consultations.

Table 4: HCW's ability to correctly identify prevalent NCDs among HIV patients

\begin{tabular}{|l|c|c|c|c|c|}
\hline \multirow{2}{*}{ Disease condition } & & \multicolumn{2}{c|}{ Yes } & \multicolumn{2}{c|}{ No } \\
\cline { 2 - 6 } & $\mathbf{N}$ & Frequency & $\%$ & Frequency & 47 \\
\hline High Blood pressure & 83 & 44 & 53 & 39 & 66.7 \\
\hline Impaired Blood sugar & 75 & 25 & 33.3 & 50 & 78.4 \\
\hline Hyperlipidaemia & 74 & 16 & 21.6 & 58 & 78.7 \\
\hline Heart Attack & 75 & 16 & 21.3 & 59 & 49.4 \\
\hline Breast Cancer & 79 & 40 & 50.6 & 39 & 92 \\
\hline Malaria & 75 & 6 & 8 & 69 & 51.9 \\
\hline Depression & 81 & 39 & 48.1 & 42 & 58.5 \\
\hline Tuberculosis & 82 & 34 & 41.5 & 48 & 4.7 \\
\hline Cervical Cancer & 86 & 82 & 95.3 & 4 & 71.1 \\
\hline Low bone mineral density & 76 & 22 & 28.9 & 54 & \\
\hline
\end{tabular}

Table 5: Behaviours screened for by HCWs during consultation with HIV infected patients

\begin{tabular}{|l|c|c|c|c|c|}
\hline \multirow{2}{*}{ Risk factor } & \multirow{2}{*}{$\mathbf{N}$} & \multicolumn{2}{|c|}{ Yes } & \multicolumn{2}{c|}{ No } \\
\cline { 3 - 6 } & Frequency & $\%$ & Frequency & $\%$ \\
\hline Smoking & 86 & 75 & 87.2 & 11 & 12.8 \\
\hline Alcohol intake & 87 & 79 & 90.8 & 8 & 9.2 \\
\hline Dietary details & 86 & 73 & 84.9 & 13 & 15.1 \\
\hline Medication adherence & 87 & 85 & 97.7 & 2 & 2.3 \\
\hline Consistent condom use & 88 & 81 & 92.0 & 7 & 8.0 \\
\hline Physical activity & 83 & 61 & 73.5 & 22 & 26.5 \\
\hline
\end{tabular}

Attitude 1: Screening HIV patients for risk factors commonly associated with NCDs is unimportant

Additionally, HCWs were asked to share their opinions on the importance of screening HIV infected patients for risk factors commonly associated with NCDs. Results show that overall $78 \%$ disagree with the statement that screening HIV patients for risk factors commonly associated with NCDs is NOT important, with $50 \%$ in strong disagreement with the statement. Only minority (11\%) believed that it is NOT important to screen HIV patients for the risk factors (Figure 3). 


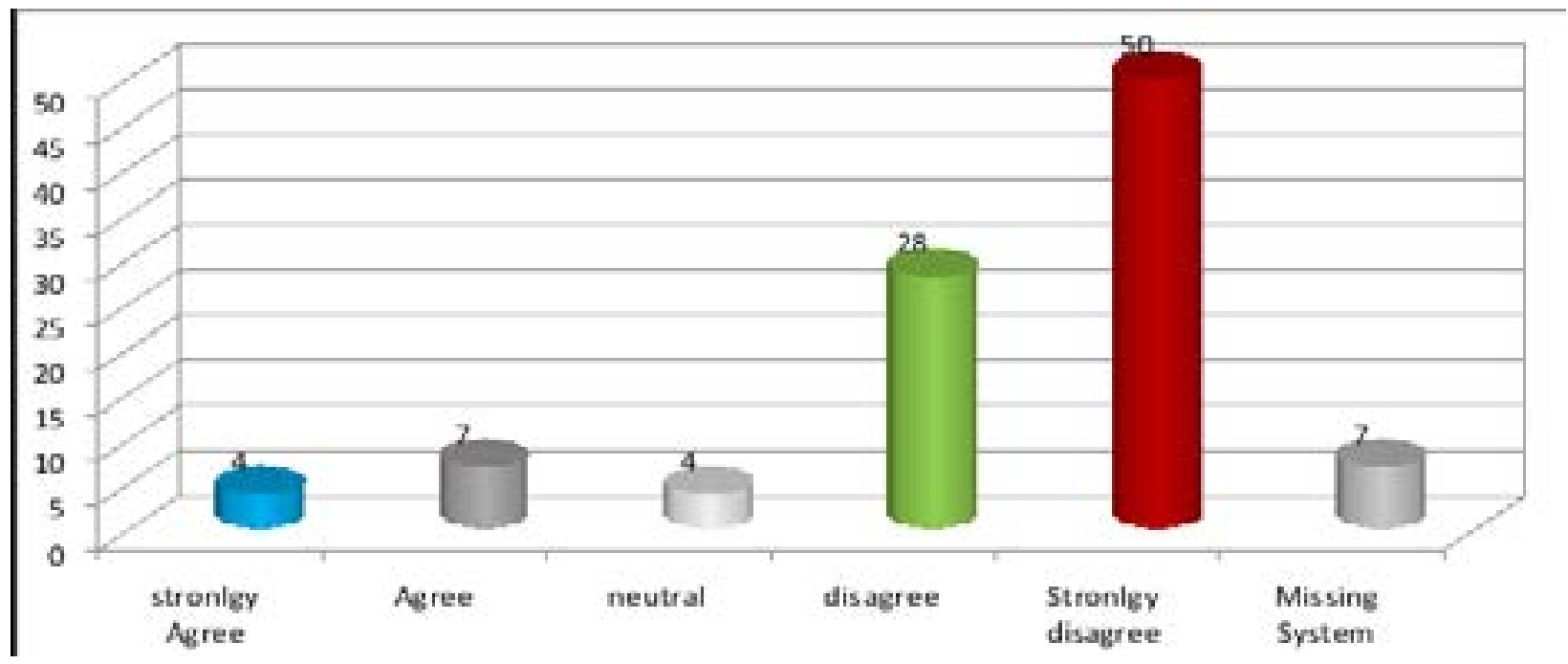

Figure 3: Attitude that screening HIV infected patients for NCDs risk factors is not important

Attitude 2: HIV patients diagnosed with NCDs should be treated at different facilities

The HCWs attitude on whether separate health facilities should be used to treat HIV patients with co-exis- tent NCDs shows that majority $72 \%$ (of which $46 \%$ did not believe and $26 \%$ did not believe at all) that the patients should be treated at separate facilities. Only $13 \%$ believed in separate treatment facilities while $7 \%$ were neutral (Figure 4).

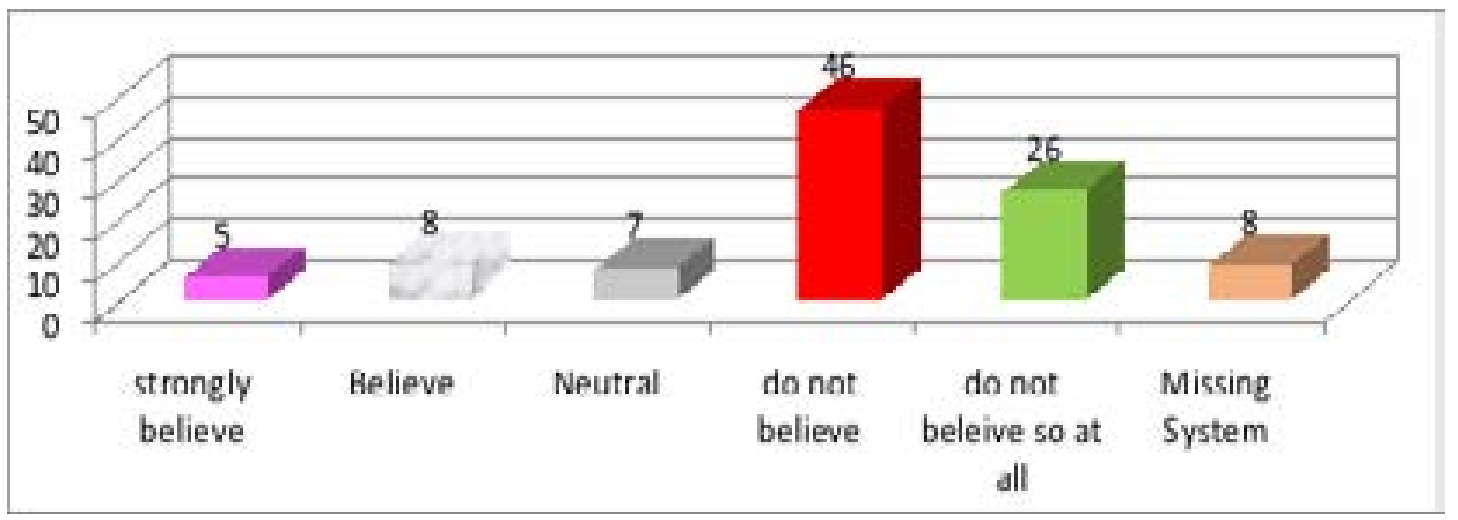

Figure 4: Whether HIV patients with co-existent NCDs should be treated at separate facilities.

Attitude 3: Integrating routine management of HIV and NCDs at primary care level (PHC)

The HCWs attitude towards integrating the routine management of HIV and NCDs at primary care level shows an overall majority of $88 \%$ (54\% strongly agree and $34 \%$ agree) to integrated routine management of HIV and NCDs at primary care level (Figure 5). Only 2\% disagreed. 


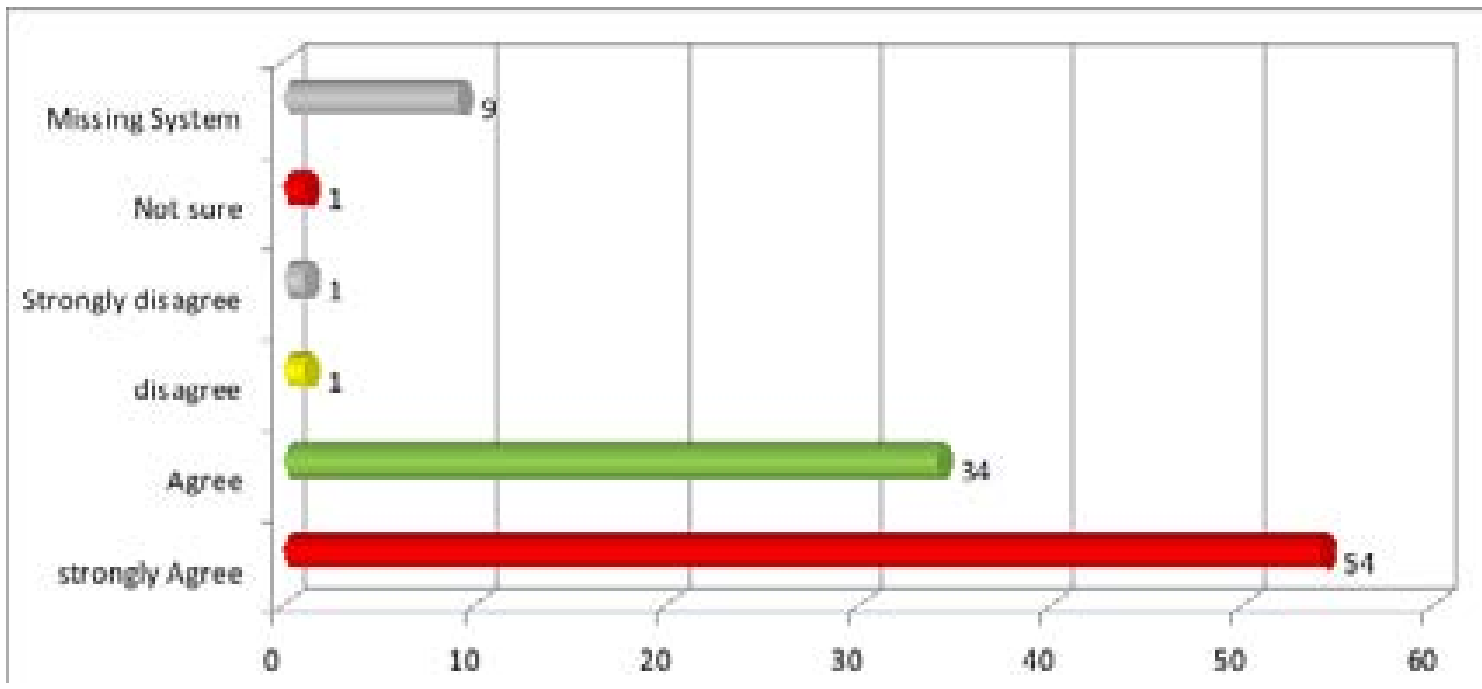

Figure 5: HCWs attitude towards integrated routine management of HIV and NCDs in PHC

\section{Attitude 4: Work load effect of integrated routine management of HIV and NCDs}

The HCWs attitude on potential work load implications of integrating NCD management into routine HIV care shows that $65.2 \%$ disagree that the integration would be additional burden to the HCWs as compared to $35 \%$ who agree that it will be a burden. Combining HIV and NCDs was perceived as good for the optimal resources utilisation as supported by $88.2 \%$ compared to $6.7 \%$ who disagreed and $4.5 \%$ who were not sure. Most respondents $(88.1 \%)$ agreed or strongly agreed that they would welcome combining of routine HIV and NCD care at primary care level.

\section{Discussion}

Urbanisation and adoption of western lifestyle have had significant health impact including growing burden of non-communicable diseases ${ }^{14,23}$ on many developing countries. In these countries health systems are being subjected to pressure because traditional infectious diseases like TB and malaria have joined with HIV/AIDS and NCDs occurring concurrently. The co-occurrence of communicable and non-communicable diseases has compelled health systems to develop innovative and cost-effective measures in health care provision. The task shifting of nurse-led clinics introduced in the sub-Saharan Africa have had positive results ${ }^{25-26}$. The WHO Framework for Innovative Care for Chronic Condition advocates for patient-centred, community inclusive collaborative health care teams which ensure that patients are informed, capacitated and motivated to own their health ${ }^{16}$. The report emphasises that health care workers during consultation should adopt motivational interviewing because such an approach has been shown to be effective in bringing about patient behavioural change $\mathrm{e}^{27-28}$.

In Southern Africa, successful rollout of ART has resulted in increased life expectancy for people living with HIV/AIDS to a point where NCDs now commonly occur $^{18}$. The chronic nature of HIV and AIDS has transformed the epidemic to a chronic disease in which the HIV patients needs are similar to those of a patient with diseases like hypertension, diabetes and cancer $^{19}$. Therefore, HIV/AIDS and NCDs are similar in terms of being primarily managed at the primary health care (PHC) level with referral to high level facility for complex cases.

The knowledge of NCDs even among highly trained practitioners has remained low. A study among internal medicine physicians in Guatemala reported that the knowledge on NCDs and its prevention was limited ${ }^{29}$. Similarly, Wellapuli \& Gunawardena ${ }^{30}$ reported in Colombo district, Sri Lanka that only $41.5 \%$ of the studied medical officers could accurately name three NCDs and as low as $37.7 \%$ were able to identify four modifiable risk factors for NCDs. In this study less than half $(48 \%)$ of the HCWs had heard about NCDs and only $6 \%$ could fully define NCDs. The ability of HCWs to identify NCDs from a list of diseases is however high suggest- 
ing that information about NCDs is accessed by HCWs from different sources. The ability of HCWs however, to correctly identify prevalent NCDs among HIV patients was varying from $95.3 \%$ cervical cancer, $33.3 \%$ impaired blood sugar metabolism and $21.6 \%$ dyslipidaemia. These findings are concerning because evidence-based reports indicate that ART increases the risk of diabetes, dyslipidaemia and myocardial infarction, information which should be easily available to $\mathrm{HCWs}{ }^{31-32}$. Additionally, the finding of about $58 \%$ and $11.8 \%$ of HCWs incorrectly identifying malaria and HIV respectively as NCDs were not expected and worrying especially with regards to HIV, a major sexually transmitted epidemic in the subSaharan Africa.

According to the WHO Global Action Plans for the Prevention and Control of NCDs for $2013-2020^{8}$, screening for NCDs is a secondary preventive approach being implemented in many countries. In this study $78 \%$ respondents strongly agree that screening for NCDs modifiable behavioural risk factors among HIV patients is important as compared to $11 \%$ who did not. Screening for medication adherence, consistent use of condom and alcohol intake were the most screened behavioural risk factors when compared with screening for smoking and dietary details. Screening for physical activity was relatively high $(73.5 \%)$ but it was lower than the other identifiable risk factors screened during consultation with HIV patients. These findings are encouraging, indicating a positive attitude and practices of HCWs towards secondary preventive measures for the prevention and control of NCDs. Some similarities with our study are observed in another resource limited setting, in Tanzania where only $14 \%$ of topics discussed with HIV patients included diabetes, 57\% smoking cessation, 64\% weight management and 86\% alcohol abuse. HIV/AIDS, child health, reproductive health, personal hygiene and nutrition were given higher priority. Therefore more work still needs to be done ${ }^{21}$. Our findings are different to those identified in a study among HIV patients accessing primary care from Partners in Health, where no differences were observed in screening rates for metabolic, cardiovascular diseases as well as cancer rates regardless of HIV care model. ${ }^{22}$. Following a successful integration of $\mathrm{HIV}$ and TB, the Botswana Government is reviewing the essential primary care package to factor in the NCD epidemic and provide comprehensive management of HIV and other chronic diseases. Providing comprehensive care requires HCWs with capacity to address all issues related to chronic diseases during single consultation and ability to interrogate effects of different co-morbid chronic diseases on the patient. Integration of NCDs and other diseases has become more topical in recent years with some different integration models suggested such as NCDs integrated with other vertical programmes such as HIV, TB, Diabetes, maternal and school health ${ }^{23}$. This is the conversation that Botswana should now be having on this subject.

\section{Conclusion}

We explored the HCW's attitudes on integrating routine NCD care with HIV management. We conclude that majority of HCWs do not prefer the current use of separate facilities to provide care to HIV patients with co-existent NCDs. In addition, most of the respondents overall don't share the view that integration of routine HIV management with NCDs would overburden them with work; and majority agree that the integration would actually optimise resources utilisation. HCWs will however, need further capacitation on NCDs management given that their level of knowledge on NCDs is inadequate and only a small proportion had ever heard about NCDs prior to this study. There were gaps in detailed knowledge on NCDs, but positive attitude towards routine primary care integrated HIV/NCDs management.

\section{Recommendations}

Various approaches to integration of services have been recommended in the literature ${ }^{33}$. We recommend a policy change which enables integrated management of NCDs and HIV at primary care level to optimise use of the limited human resource for health. The current national strategy for NCDs 2017-2022 in Botswana is proposing such integration of HIV and NCD programs. Any proposed integration of care for the people with NCDs and those receiving ART however, should be a well thought out planned process implemented in stages with necessary key stakeholder consultation (HCWs and patients) during development of the relevant guidelines. Training of HCWs should include epidemiology and pathophysiology of NCDs in general, NCDs specific to HIV/ AIDS and motivational history taking by HCWs during consultation with patients. The training could be through formal mainstreaming of the concepts of integrated care models to undergraduate and graduate medical and nurs- 
ing education, regular in-service short courses and continuing professional development. Community mobilization against NCDs should be intensified with advocacy for early detection of NCDs.

\section{Limitations}

The main limitation of the findings in this study is the small sample size. However, the sample was collected from Gaborone where most of health care workers are found in Botswana and it is in this region where most information related to health can easily be accessed by practitioners.

\section{Acknowledgements}

The authors wish to extend appreciation to all HCWs for their time and valuable information. This paper is based on a project supported by Afya Bora Consortium Fellowship, which is funded by the President's Emergency Plan for AIDS Relief (PEPFAR) and the Office of AIDS Research (OAR) of the U.S. National Institutes of Health through funding to the University of Washington under Cooperative Agreement U91 HA06801 from the US Department of Health and Human Services, Health Resources and Services Administration (HRSA) Global HIV/AIDS Bureau.

\section{Conflict of interest}

None to declare.

\section{References}

1. World Health Organisation. HIV/AIDS [Internet]. Global Health Observatory (GHO) Data. 2015 [cited 2017 Jan 26]. Available from: http://www.who.int/gho/ hiv/en

2. NACA. Progress Report of the National Response to the 2011 Declaration of Commitments on HIV and AIDS. 2015;(June):34. Available from: http://www.unaids.org/sites/default/files/country/documents/BWA_ narrative_report_2015.pdf

3. Dorrington, R E. Moultrie, T A. Daniel T. The demographic impact of HIV/AIDS in Botswana. NACA, editor. Gaborone; 2006. 169 p.

4. World Health Organization (Who). Country Health Profile Botswana [Internet]. [cited 2017 Jan 26]. Available from: http://www.who.int/countries/bwa/en/
5. Reid, M. Tsima, B. Kirk B. HIV and diabetes in Africa. African J Diabetes Med. 2012;20(2):28-32.

6. World Health Organisation. Global Action Plan for the prevention and control of non-communicable diseases 2013 - 2020. Geneva; 2013.

7. Fuster, V. Kelly, BB. Vedanthan R. Promoting global cardiovascular health moving forward. Circulation. 2011;123(15):1671-8.

8. Ministry of Health Botswana. Strategic plan for prevention and control of non-communicable diseases 20112016. Gaborone; 2011.

9. Ministry of Health Republic of Botswana World Health Organization. Chronic disease risk factor surveillance report. Gaborone; 2007.

10. Ministry of Health Botswana. Health Statistics Report. Gaborone; 2009.

11. Abegunde, DO. Mathers, CD. Adam. T. Ortegon, M. Strong K. The burden and costs of chronic diseases in low-income and middle-income countries. Lancet. 2007;370(9603):1929-38.

12. Oni T, McGrath N, BeLue R, Roderick P, Colagiuri S, May CR, et al. Chronic diseases and multi-morbidity - a conceptual modification to the WHO ICCC model for countries in health transition. BMC Public Health [nternet]. 2014;14(1):575. Available from: http://www. biomedcentral.com/1471-2458/14/575\%5Cnhttp:// www.pubmedcentral.nih.gov/articlerender.fcgi?artid $=4071801 \&$ tool $=$ pmcentrez\&rendertype $=$ abstract

13. Health $\mathrm{M}$ of. Health service Package for Botswana The Essential The Essential Health Service Package for Botswana. Gaborone; 2010.

14. Lopex A. Disease control priorities: Global burden of diseases and risk factors [Internet]. New York; 2006. Available from: www.dcporg

15. Callaghan, M. Ford, N. Schneider H. A systematic review of task shifting for HIV treatment and care in Africa. Hum Resour Heal. 2010;8(8).

16. World Health Organisation. Innovative Care for Chronic Conditions. World Health. Geneva; 2002.

17. Anstiss T. Motivational interviewing in primary care. J Clin Psychol Med Settings. 2009;16:87-93 PubMed .

18. Levitt, N. Bradshaw D. The impact of HIV/AIDS on type 2 diabetes prevalence and diabetes health care needs in South Africa: projections for 2010. Diabet Med. 2006;23:103-4 PubMed. 
19. Levitt NS, Steyn K, Dave J, Bradshaw D. Chronic noncommunicable diseases and HIV-AIDS on a collision course: Relevance for health care delivery, particularly in low-resource settings - Insights from South Africa. Am J Clin Nutr. 2011;94(6):1690 PubMed -6.

20. Samaras K. Metabolic consequences and therapeutic options in highly active antiretroviral therapy in human immunodeficiency virus-1 infection. I Antimicrob Chemother. 2008;61:238-245.

21. Leung C, Aris E, Mhalu A, Siril H, Christian B, Koda $\mathrm{H}$, et al. Preparedness of HIV care and treatment clinics for the management of concomitant non-communicable diseases: A cross-sectional survey. BMC Public Health [nternet]. 2016;16(1):1-8. Available from: http://dx.doi. org/10.1186/s12889-016-3661-1

22. Rhodes CM, Chang Y, Regan S, Triant VA. Non-Communicable Disease Preventive Screening by HIV Care Model. PLoS One. 2017;12(1):1 PubMed -13.

23. Narain JP. Integrating Services for Noncommunicable Diseases Prevention and Control: Use of Primary Care Approach. Indian J community Med. 2011;36(Supp 1 S67-S71). 